\title{
Punctate white matter lesions in infants: new insights using susceptibility-weighted imaging
}

\author{
Tetsu Niwa $\cdot$ Linda S. de Vries • \\ Manon J. N. L. Benders • Taro Takahara • \\ Peter G. J. Nikkels • Floris Groenendaal
}

Received: 22 October 2010 /Accepted: 11 April 2011 /Published online: 7 May 2011

(C) The Author(s) 2011. This article is published with open access at Springerlink.com

\begin{abstract}
Introduction Punctate white matter lesions (PWML) are recognized with magnetic resonance imaging (MRI) as hypersignal on T1-weighted imaging and hyposignal on T2-weighted imaging. Our aim was to assess how often a hemorrhagic component was present in PWML using susceptibility-weighted imaging (SWI).

Methods Seventeen preterm (gestational age, 25-34 weeks) and seven full-term infants (age at MRI, 37-42 weeks) with PWML were included. Seven preterm infants had sequential MRIs. PWML were diagnosed with conventional MRI and compared with SWI, where signal loss is suggestive of hemorrhage. The pattern of associated brain lesions was taken into account, and the percentage of lesions with signal loss on SWI was calculated for each infant.

Results A significantly higher percentage of signal loss on
\end{abstract}

Electronic supplementary material The online version of this article (doi:10.1007/s00234-011-0872-0) contains supplementary material, which is available to authorized users.

T. Niwa $\cdot$ T. Takahara

Department of Radiology, University Medical Center Utrecht, Heidelberglaan 100, PO Box 85500, 3508 GA Utrecht,

The Netherlands

L. S. de Vries • M. J. N. L. Benders • F. Groenendaal

Department of Neonatology, Wilhelmina Children's Hospital/

University Medical Center Utrecht,

KE 04.123.1, PO Box 85090, 3508 AB Utrecht, The Netherlands

P. G. J. Nikkels

Department of Pathology, University Medical Center Utrecht, Heidelberglaan 100, PO Box 85500, 3508 GA Utrecht,

The Netherlands

T. Niwa $(\square)$

Departments of Radiology, Kanagawa Children's Medical Center,

2-138-4 Mutsukawa, Minami-ku,

Yokohama 232-8555, Japan

e-mail: tniwa@kcmc.jp
SWI (median, 93.9\%) was found among infants with germinal matrix and intraventricular hemorrhage as the primary diagnosis $(n=8)$ compared to those with a primary diagnosis of white matter injury ( $n=14$; median, $14.2 \%$; $p<0.01)$. In the infants with serial MRIs, a reduction in the number of PWML and/or signal loss on SWI was noted at term equivalent age. In the patient who died, cystic lesions, associated with hemorrhage and gliosis, were demonstrated on histology.

Conclusions SWI can distinguish hemorrhagic and nonhemorrhagic PWML. Signal loss on SWI was more common when PWML were associated with an intraventricular hemorrhage. Longitudinal imaging showed a decrease in the number of PWML over time, with some PWML no longer showing signal loss on SWI, suggesting early gliosis.

Keywords Punctate white matter lesions - Neonate Susceptibility-weighted imaging $\cdot$ Hemorrhage
Abbreviations
PWML Punctate white matter lesions
SWI Susceptibility-weighted imaging
MRI Magnetic resonance imaging
IR Inversion recovery
SENSE Sensitivity encoding
TEA Term equivalent age
PMA Postmenstrual age

\section{Introduction}

With the increasing use of magnetic resonance imaging (MRI), hemorrhagic and hypoxic-ischemic lesions are often diagnosed during the neonatal period. Punctate white 
matter lesions (PWML) are a common finding in preterm infants [1-7]. PWML can be recognized on MR images in the unmyelinated white matter as hypersignal on T1weighted imaging and hyposignal on T2-weighted imaging. These signal intensity changes can be compatible with either petechial hemorrhages, gliotic scarring, or mineralization, which are impossible to distinguish on T1- and T2weighted images $[1,2,6,8,9]$. Most preterm infants with these PWML survive, and detailed data on postmortem histology are scarce [10]. Histology has demonstrated that petechial hemorrhages are seen in about $25 \%$ of infants with white matter lesions [11]. More recently, it has been shown that white matter injury does not occur exclusively in preterm infants, and attention has especially been drawn to full-term infants with congenital heart defects undergoing surgery in the neonatal period [1, 12-14].

Susceptibility-weighted imaging (SWI) has recently been developed for clinical use. This sequence is very sensitive to detect susceptibility changes such as blood, iron, calcification, and air. SWI uses a high-spatial resolution, three-dimensional, fast low-angle shot MRI technique with postprocessing with multiplying phase data [15-17]. Recent studies have shown that SWI is superior to gradient echo sequence or CT in detecting hemorrhage [1820]. Detection of hemorrhagic components is useful and will help to understand the neuropathological conditions underlying the signal intensity changes seen on MRI.

The aim of this study was to assess whether SWI can help to make a distinction between hemorrhagic and ischemic PWML, seen on MRI in the neonatal period. In addition, the evolution of these lesions was assessed in a subgroup of infants examined sequentially.

\section{Materials and methods}

\section{Patients}

Between June 2009 and September 2010, SWI sequences were obtained in infants, who were scanned for clinical reasons. SWI sequences were added to the routine scanning protocol. The study was approved by the institutional review board, and parental informed consent was obtained. Infants were eligible for this study when PWML were present, seen as hypersignal on T1-weighted or inversion recovery (IR) imaging and hyposignal on T2-weighted imaging in the unmyelinated white matter.

During this period, an MRI was performed in 59 preterm infants, and PWML was diagnosed in 15 (25.4\%). A further 105 full-term infants were examined with a variety of underlying problems, the majority because of hypoxic ischemic encephalopathy, and six $(5.7 \%)$ were found to have PWML. Four infants with white matter abnormality due to venous thrombosis $(n=2)$, vein of Galen malformation $(n=1)$, and neuromuscular disorder $(n=1)$ were excluded because of potentially different etiologies. One MR examination performed at 33 weeks postmenstrual age (PMA) could not be used due to the presence of severe motion artifacts, but the MR examination of this infant obtained at term equivalent age (TEA) was included. Finally, 17 preterm infant with a gestational age of 25 34 weeks (median gestational age, 31 weeks; median birth weight, $1541 \mathrm{~g}$ ) and seven full-term infants were included. Twelve of the preterm infants were scanned in the immediate neonatal period, and five of the preterm infants were first examined at TEA. Seven preterm infants were examined in the immediate neonatal period and at TEA.

\section{MRI}

MRI was performed using either a 3- or 1.5-T magnet (Achieva or ACS-NT; Philips Healthcare, Best, The Netherlands). Coils for scanning were chosen as follows: a dedicated eight-channel sensitivity encoding (SENSE) head coil for full-term infants or preterm infants who had an MRI at TEA, or a combination of four surface coils for preterm infants who had an MRI at 30-32 weeks PMA using a dedicated MR-compatible incubator [21]. All neonates were sedated according to the method described previously using oral chloral hydrate or a combination of pethidine, chlorpromazine and promethazine i.m. [22]. Hearing protection (Natus Medical Incorporated, San Carlos, CA, USA) was used in all neonates. A vacuum pillow (Med-Tec, Orange City, IA, USA) was used to prevent excessive head movement and for additional hearing protection. Intensive care was continued during the examination, and heart rate and transcutaneous oxygen saturation were monitored continuously by pulse oximetry (Nonin, Mineapolis, MN, USA) in all infants as well as respiration rate in the infants with spontaneous respiration. When needed, infants received mechanical ventilation, using a BabyPAC 100 (Pneupac Ltd, Luton, UK).

Routine MRI on a 3-T scanner included a transverse or coronal T2-weighted fast spin-echo imaging [repetition time/echo time, 6,291/120 ms; echo train length (ETL), 15; matrix $293 \times 263$; slice thickness, $2 \mathrm{~mm}$; two signals acquired], a transverse or coronal T1-weighted 3Dgradient-echo image (495/10; flip angle, $8^{\circ}$; matrix, 192× 192; slice thickness, $2 \mathrm{~mm}$; six signals acquired), a sagittal T1-weighted spin-echo image $(885 / 15$; matrix, $205 \times 256$; slice thickness, $3 \mathrm{~mm}$; four signals acquired); those on a 1.5-T scanner included a transverse $\mathrm{T} 2$-weighted fast spinecho image $(7820 / 150$; ETL 21 ; matrix $237 \times 256$; slice thickness, $2 \mathrm{~mm}$; two signals acquired), a transverse IR image (4,146/30; inversion time, $600 \mathrm{~ms}$; matrix, $218 \times 256$; ETL, 3; slice thickness, $4 \mathrm{~mm}$; seven signals acquired), a 


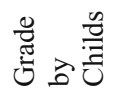

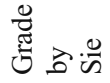

誉言

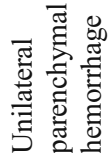

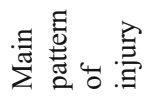

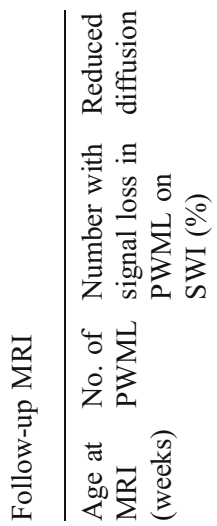

럴류

$\cong$

象 0

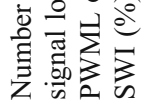

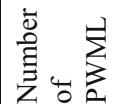

.5

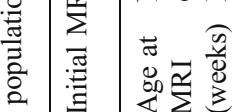

줗

$\stackrel{\square}{g}$

要

藏

范

焉

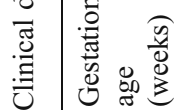

U 0

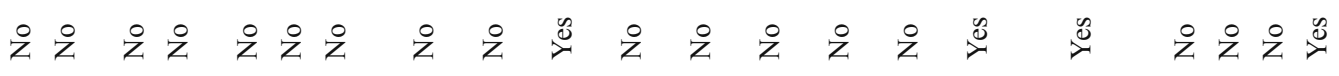

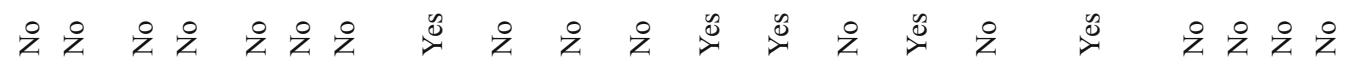

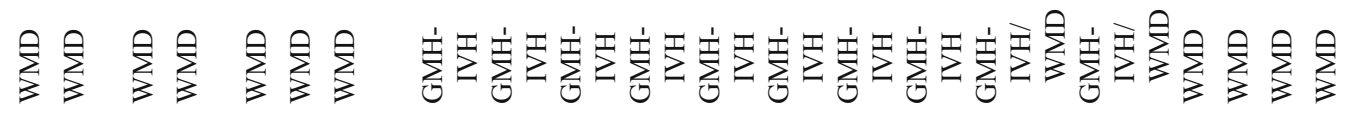

そ̊ そ

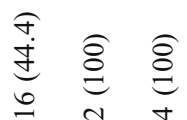

¿

$\cong \simeq$

$\therefore \simeq$

₹ ช

2 2

भ ₹

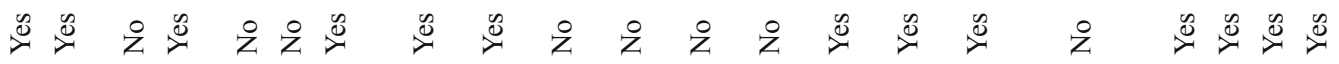

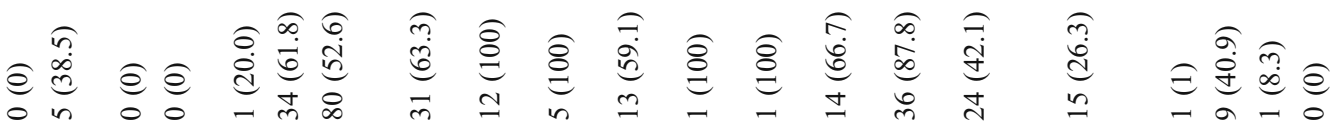

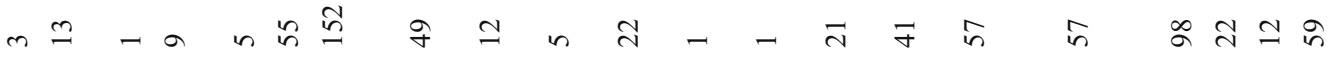

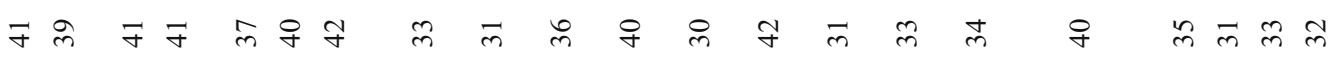

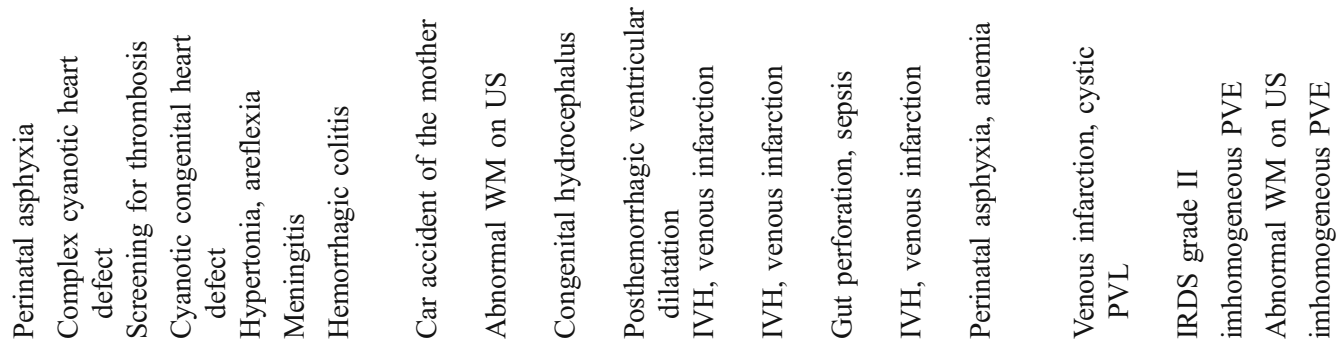
茀

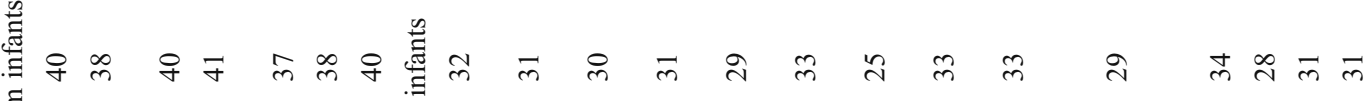

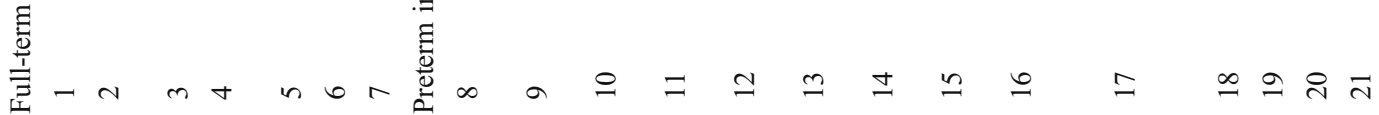


sagittal T1-weighted spin-echo image (512/15; matrix, $154 \times 256$; slice thickness, $4 \mathrm{~mm}$; one signal acquired), field of view (FOV) was set to $130-200 \mathrm{~mm}$ depending on patients' head size and imaging plane. Diffusion-weighted MRI was part of the standard MRI protocol ( $b$ value, $1,600 \mathrm{~s} / \mathrm{mm}^{2}$ at $3 \mathrm{~T} ; 1,000 \mathrm{~s} / \mathrm{mm}^{2}$ at $\left.1.5 \mathrm{~T}\right)$.

SWI was performed using a 3D gradient-echo sequence with flow compensation. Since it is important to keep the scan time as short as possible to minimize the chance of motion artifacts in neonatal imaging, multishot echo-planar imaging (EPI) was employed in the SWI sequence in addition to parallel imaging. The sequence parameters were as follows: at $3 \mathrm{~T}$, TR/TE is $52 / 30 \mathrm{~ms}$, SENSE factor is 1.7 , total acquisition time is $2 \min 33 \mathrm{~s}$; at $1.5 \mathrm{~T}, \mathrm{TR} / \mathrm{TE}$ is $82 / 40 \mathrm{~ms}$, SENSE factor is 2 , total acquisition time is $2 \min 53 \mathrm{~s}$. Other parameters were the same at both 3 and $1.5 \mathrm{~T}$, as follows: flip angle, $15^{\circ}$; FOV, $160 \times 144 \mathrm{~mm}$; matrix, $320 \times 289$; section thickness, $2 \mathrm{~mm}$; slab thickness, $80 \mathrm{~mm}$; bandwidth, $36.2 \mathrm{~Hz} /$ pixel; and EPI factor, 3. Using zero-filling technique, images were reconstructed with a $512 \times 512$ matrix and a $1-\mathrm{mm}$ slice interval. For creating SWI, image data were transferred to on offline PC. With an in-house program, filtering and multiplication of phase data and minimum intensity projection were done according to the technique described previously $[16,17,23]$. Finally, SWI was displayed in the transverse plane using 4-mm thick contiguous slices.

Data analysis

When T1-weighted images were not obtained with the same angulations as SWI, they were reconstructed on a workstation (Philips Healthcare, Best, The Netherlands) to be able to make a comparison between the different sequences.

Using conventional images (T1-weighted/IR and T2weighted images) a diagnosis was made of the main pattern of injury:

1. Germinal matrix hemorrhage/intraventricular hemorrhage (GMH-IVH) with or without a unilateral parenchymal hemorrhage (classified according to Papile et al.) [24].

2. Subtle to severe white matter damage (WMD), with or without cystic evolution, depending on the time of the MRI (classified according to de Vries et al.) [25].

PWML were subsequently assessed by two authors experienced in neonatal neuroimaging ( $\mathrm{TN}$ and $\mathrm{FG}$ ). Consensus about the presence and number of PWML was reached in all cases. PWML were defined as small areas of hypersignal on T1-weighted images and hyposignal on T2weighted images. Signal intensity changes within cystic lesions were excluded from the analysis. In infants with a unilateral parenchymal hemorrhage [26, 27], only the PWML 
Fig. 1 Punctate white matter lesions in a preterm infant born at gestational age of 31 weeks, scanned on day 3 (case 9): GMH-IVH pattern. a T1weighted image shows punctate hypersignal lesions in the white matter (arrows). b SWI at the corresponding slice to a shows signal loss at the punctate hypersignal lesions on T1weighted image, suggesting the presence of hemorrhage (arrows). c T1-weighted image at the level of the basal ganglia shows germinal matrix hemorrhage (arrows) and intraventricular hemorrhage (arrowhead) as hypersignal lesions. d SWI at the corresponding slice to $\mathbf{c}$ hemorrhage (arrows) and intraventricular hemorrhage (arrowhead) shows bilateral germinal matrix
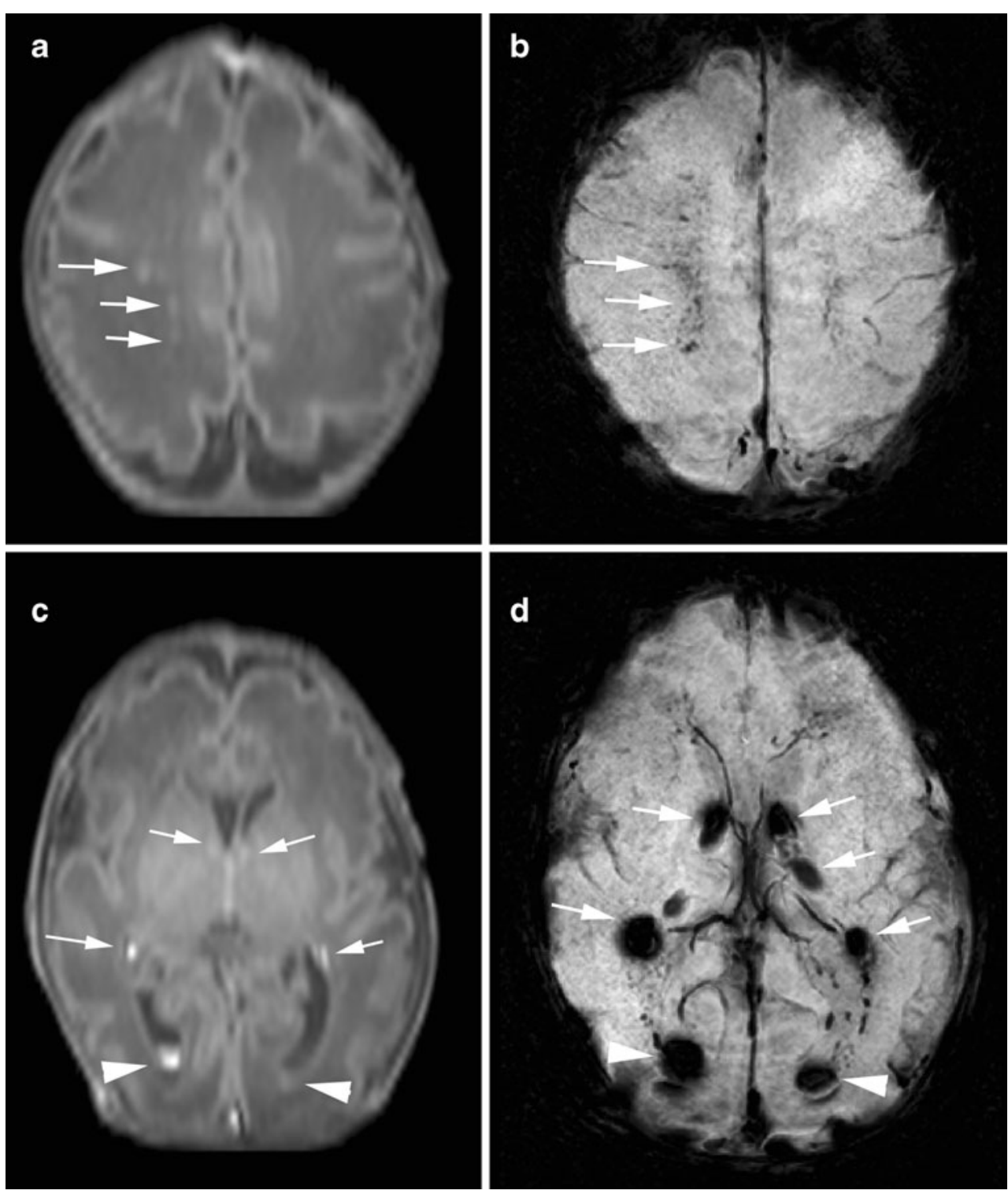

Fig. 2 Punctate white matter lesions in a full-term neonate with perinatal asphyxia, scanned on day 4 (case 1): WMD pattern. a IR image shows nodular and dotted hypersignal lesions in the white matter (arrows). b SWI at the corresponding slice to a shows no signal loss that would represent hemorrhage in the white matter lesions that would suggest the presence of hemorrhage
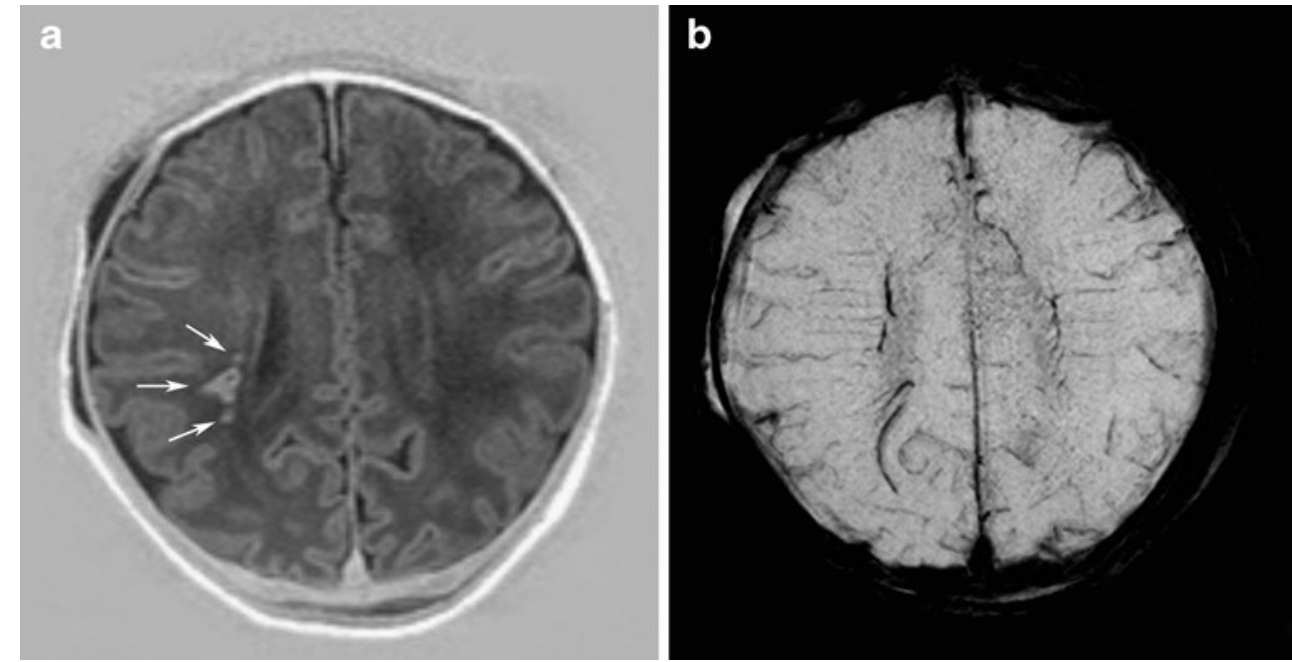
were analyzed that were outside the immediate area of the parenchymal hemorrhage. First, T1-weighted or IR images were reviewed with respect to the location and number of PWML. Areas of myelination seen as areas of hypersignal were not included [28, 29]. Following this analysis, the presence or absence of signal loss in the PWML on corresponding SWI was scored at the corresponding location to PWML. Foci of signal loss without continuity suggestive of veins on SWI were interpreted as hemorrhage. When the deep veins were dilated, foci of signal loss larger than the surrounding veins were considered as hemorrhage. Cystic evolution was noted when areas with signal intensity identical to that of cerebrospinal fluid on all sequence were found in the white matter.

PWML were graded by the scoring system described by Sie et al. [9] and Childs et al. [1]. In seven cases with serial MRIs, the number of PWML was calculated for both MR images as well as the number of PWML with signal loss on SWI.

Apparent diffusion coefficient maps were subsequently generated on MRI unit's software. PWML were analyzed with regard to showing hypersignal on diffusion-weighted imaging and hyposignal on ADC map, in comparison to the surrounding white matter.

\section{Histology}

In the patient who died (case 23), histology was performed as described previously [30]. Brain tissue obtained at postmortem examination was fixed using $4 \%$ buffered paraformaldehyde for 4 weeks. Samples for histology were taken from different brain regions, including periventricular white matter. The samples were embedded in paraffin by standard histological procedures. Histological sections were cut at 5-6 $\mu \mathrm{m}$ and were mounted on coated slides. Tissue was stained with hematoxylin-eosin (Klinipath, Duiven, The Netherlands). Before staining with antibodies, slides were placed in boiling citrate buffer ( $\mathrm{pH}$ 6.0) for $20 \mathrm{~min}$. To study glial fibrillary acidic protein (GFAP), sections were stained with mouse monoclonal anti human glial fibrillary acidic protein antibodies, clone 6F2 (dilution, 1:3,200; DakoCytomation Denmark) and counterstained with hematoxylin. Monoclonal mouse antibodies against CD68/ED1 (Novo Castra, Newcastle, UK) were used to determine the presence of activated microglia and macrophages. Negative controls were created after omission of the specific (primary) antibody, and no staining was observed. Areas of GFAPpositive cells were considered early gliosis. Areas of GFAP-positive cells were compared to MRI findings.

\section{Statistical analysis}

To assess the relation between imaging factors and the percentage of signal loss on SWI in PWML, the percentage of signal loss on SWI in the initial MRI were compared between infants grouped with following factors using independent sample $t$ test: gestational age (preterm or term infant), age at MRI [early (e.g., MRI at 30-36 weeks), or TEA (e.g., MRI at 37-43 weeks)], main pattern of injury (GMH-IVH or WMD), presence or absence of unilateral parenchymal hemorrhage, presence or absence of cystic evolution, and scoring system. Statistical analyses were performed by using Medcalc version 10.4.5.0 software (MedCalc Software, Mariakerke, Belgium). Differences with $p<0.05$ were considered statistically significant.

\section{Results}

Table 1 shows a summary of the clinical and imaging data regarding gestational age, age at MR scanning, the 8number

Table 2 Comparison of clinical and imaging factors to hemorrhagic rate on SWI in white matter lesions

\begin{tabular}{|c|c|c|c|}
\hline Variables & $\begin{array}{l}\text { Number of } \\
\text { cases }\end{array}$ & $\begin{array}{l}\text { Median percentage } \\
\text { of hemorrhage in } \\
\text { white matter } \\
\text { lesions }(\%)\end{array}$ & $p$ value \\
\hline \multicolumn{4}{|c|}{ Gestational age (pre-/full-term) } \\
\hline Preterm & 17 & 59.1 & \multirow[t]{2}{*}{0.06} \\
\hline Full-term & 7 & 20.0 & \\
\hline \multicolumn{4}{|c|}{ Age at MRI (early/TEA) } \\
\hline Early & 12 & 64.9 & \multirow[t]{2}{*}{0.13} \\
\hline TEA & 12 & 32.3 & \\
\hline \multicolumn{4}{|c|}{ Main pattern of injury ${ }^{a}$} \\
\hline GMH-IVH & 8 & 93.9 & \multirow[t]{2}{*}{$<0.01$} \\
\hline WMD & 14 & 14.2 & \\
\hline \multicolumn{4}{|c|}{ Unilateral parenchymal hemorrhage } \\
\hline Presence & 5 & 87.8 & \multirow[t]{2}{*}{0.05} \\
\hline Absence & 19 & 40.9 & \\
\hline \multicolumn{4}{|c|}{ Cystic evolution } \\
\hline Presence & 6 & 48.8 & \multirow[t]{2}{*}{0.72} \\
\hline Absence & 18 & 46.7 & \\
\hline \multicolumn{4}{|l|}{ Grade by Sie } \\
\hline Grade 3 & 5 & 0.0 & \multirow[t]{2}{*}{0.13} \\
\hline Grade 4 & 19 & 55.6 & \\
\hline \multicolumn{4}{|c|}{ Grade by Childs } \\
\hline Grade 1 or 2 & 14 & 39.7 & \multirow[t]{2}{*}{0.59} \\
\hline Grade 3 or 4 & 10 & 54.1 & \\
\hline
\end{tabular}

TEA term equivalent age, GMH-IVH germinal matrix hemorrhage/ intraventricular hemorrhage, $W M D$ subtle to severe white matter damage

${ }^{\mathrm{a}}$ Two infants was excluded for analysis because equal distribution of GMH-IVH and WMD was found 
of PWML, the number and percentage of PWML with signal loss on SWI, main pattern of injury (GMH-IVH or WMD), unilateral intraparenchymal hemorrhage, cystic evolution in the white matter, and grade of the white matter lesions.

\section{GMH-IVH pattern}

Eight infants had a GMH-IVH, and in four of them, there was an associated unilateral parenchymal hemorrhage. All infants with a GMH-IVH were born preterm. Six infants had an MR examination at an early PMA (e. g., 30-36 weeks), two at TEA, and three infants on both occasions. The median number of PWML was 16.5 (range, 1-49), and the median percentage of signal loss in PWML using SWI was $93.9 \%$ (range, from $59.1 \%$ to $100 \%$, Fig. 1). Most PMWLs were seen close to the medullary veins. Four infants showed reduced diffusion on their early MRI.

\section{WMD pattern}

Fourteen infants had WMD, and in three of them, the MRI showed cystic evolution [cystic periventricular leukomalacia (PVL) grade III]. Seven of the infants were born preterm, and seven infants were born at term. Five infants had an early MRI performed at a PMA (e.g., 32-35 weeks), and nine infants had an MRI at TEA. Two of these infants had an MRI on both occasions. The median number of PWML was 12.5 (range, 1-163), and median percentage of signal loss in PWML using SWI was $14.2 \%$ (range from $0 \%$ to $81.6 \%$; Fig. 2). Five preterm infants and four fullterm infants at an early MRI showed reduced diffusion.
Fig. 3 Punctate white matter lesions in a preterm infant born at gestational age of 32 weeks born following an emergency cesarean section following a serious car accident of the mother. MRI was performed at 33 and 43 weeks postmenstrual. To manage post-hemorrhagic hydrocephalus a subcutaneous reservoir was placed after the initial scan (case 8). a Initial T1weighted image shows multiple hypersignal lesions with dotted, nodular and patchy shapes in the white matter (arrows). b Initial SWI at the corresponding slice to a shows signal loss in many of the PWML (arrows). Diffuse signal loss in the lateral ventricles represents large amount of intraventricular hemorrhage (arrowheads). c Follow-up T1weighted image shows reduced hypersignal in the PWML (arrows). d Follow-up SWI at the corresponding slice to $\mathbf{c}$ shows decreased rate of signal loss in PWML (arrows). Note that some of the signal loss at SWI that was visible in the initial scan is no longer seen on the follow-up scan
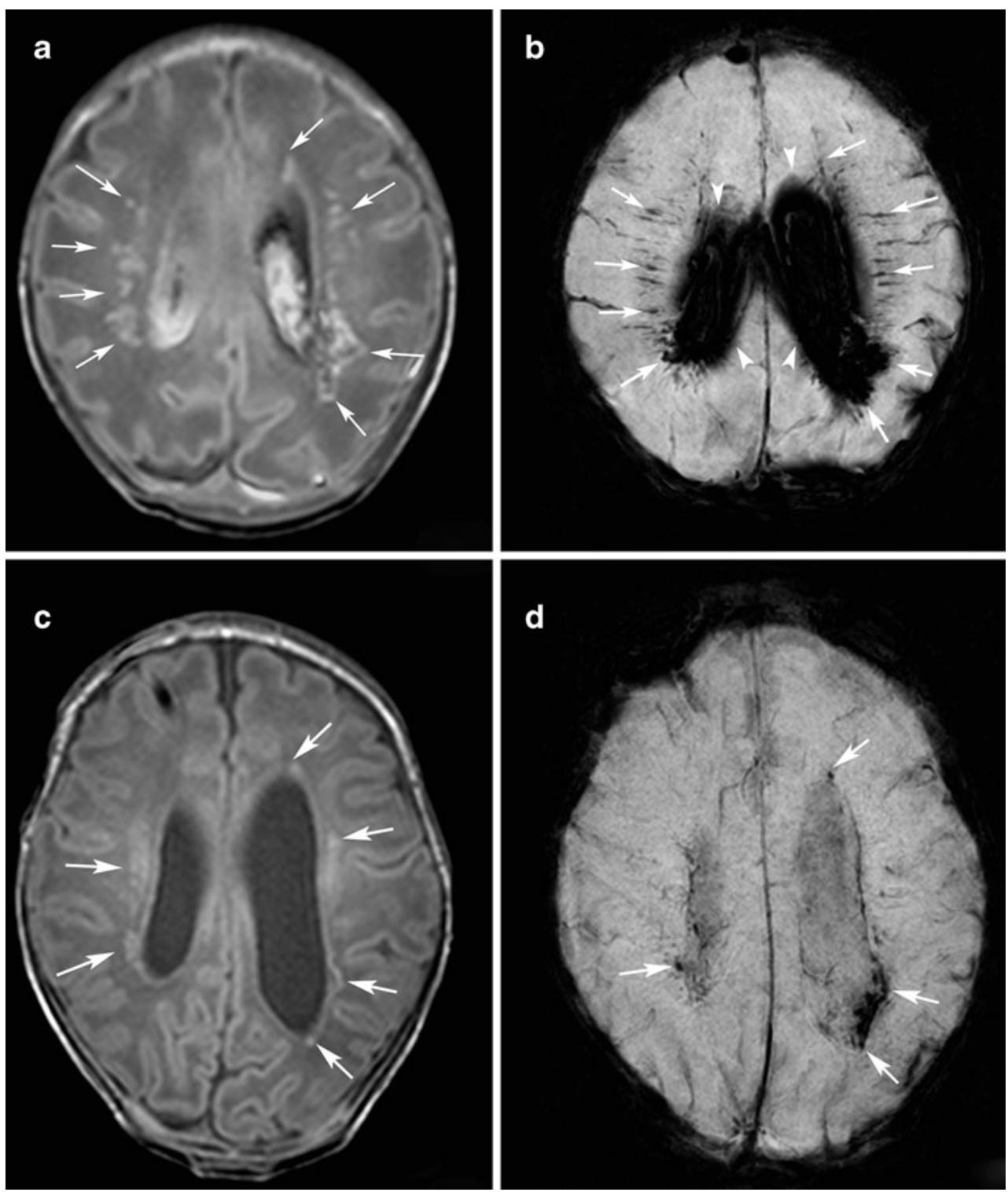


\section{Mixed GMH-IVH and WMD pattern}

In two preterm infants, there was a combination of GMHIVH and WMD. One had both a venous infarction as well as cystic PVL. One of them showed reduced diffusion at an early MRI.

Comparison of the imaging data and percentage of signal loss on SWI in PWML

Table 2 shows a summary of the comparison. The percentage of signal loss on SWI was significantly higher in infants with GMH-IVH, compared with infants with WMD (median, 93.9\%, 14.2\%, respectively; $p<0.01$ ). On early MRI (e.g., 30-36 weeks PMA), 1-49 PWML were seen in six infants with GMH-IVH, compared to 12-163 PWML for five infants with WMD. On the TEA MRI, 122 PWML were seen in two infants with GMH-IVH and 1152 PWML in nine infants with WMD. The percentage of signal loss on SWI in PWML was not significantly higher on the early MRI. There was a trend toward a higher percentage of signal loss on SWI in PWML in preterm infants compared to full-term infants $(p=0.06)$ and infants with a unilateral parenchymal hemorrhage $(p=0.05)$. The percentage of signal loss on SWI was not significantly different between the infants with or without cystic evolution or infants grouped with scoring system by Sie et al. [9] and Childs et al. [1].

\section{Change in PWML on sequential MRI}

Seven infants had an MRI in the immediate neonatal period and at TEA (Table 1). A decrease in the number of PWML was seen in all seven patients (Fig. 3). The number of punctate lesions showing signal loss on SWI decreased with time in six of these seven infants. In one infant, signal loss on SWI in PWML detected on the early MRI was noted to persist at TEA MRI.

\section{Histology}

Results of the postmortem examination are presented in Figs. 4 and 5. Hemorrhagic periventricular leukomalacia was confirmed macroscopically, whereas histology demonstrated many areas of GFAP-positive cells indicating early gliosis in areas of brain tissue with abnormalities on T1-weighted imaging, but no signal loss on SWI.

\section{Discussion}

In this study, we were able to make a distinction between hemorrhagic and non-hemorrhagic PWML using SWI. Infants with a primary diagnosis of GMH-IVH and associated PWML, more often had areas of signal loss on SWI, suggestive of petechial hemorrhages, compared to those with a primary diagnosis of WMD. These findings would suggest that PWML were more often hemorrhagic when associated with GMH-IVH and more often ischemic in those with WMD. The reason of a higher rate of hemorrhagic components in PWML, as detected with SWI, in infants with GMH-IVH may be explained by the fact that in patients with GMH-IVH, both impaired venous drainage and elevation of the pressure in the medullary veins occur in the periventricular white matter [26, 27], resulting in a higher occurrence of hemorrhage. Most hemorrhagic PWML were seen close to veins. Previous studied have reported PWML in preterm infants who had early MRI (30-32 weeks PMA) as well as in preterm infants scanned at TEA. Non-hemorrhagic PWMLs were
Fig. 4 Punctate white matter lesions in a preterm infant born at gestational age of 31 weeks, scanned on day 11. Infant died at 13 days of age (case 23). a T1-weighted image shows punctate hypersignal lesions (arrows) and cystic areas (arrowheads) in the white matter. b SWI at the corresponding slice to a shows signal loss at the punctate hypersignal lesions and cystic areas on T1-weighted image, suggesting the presence of hemorrhage (arrows)
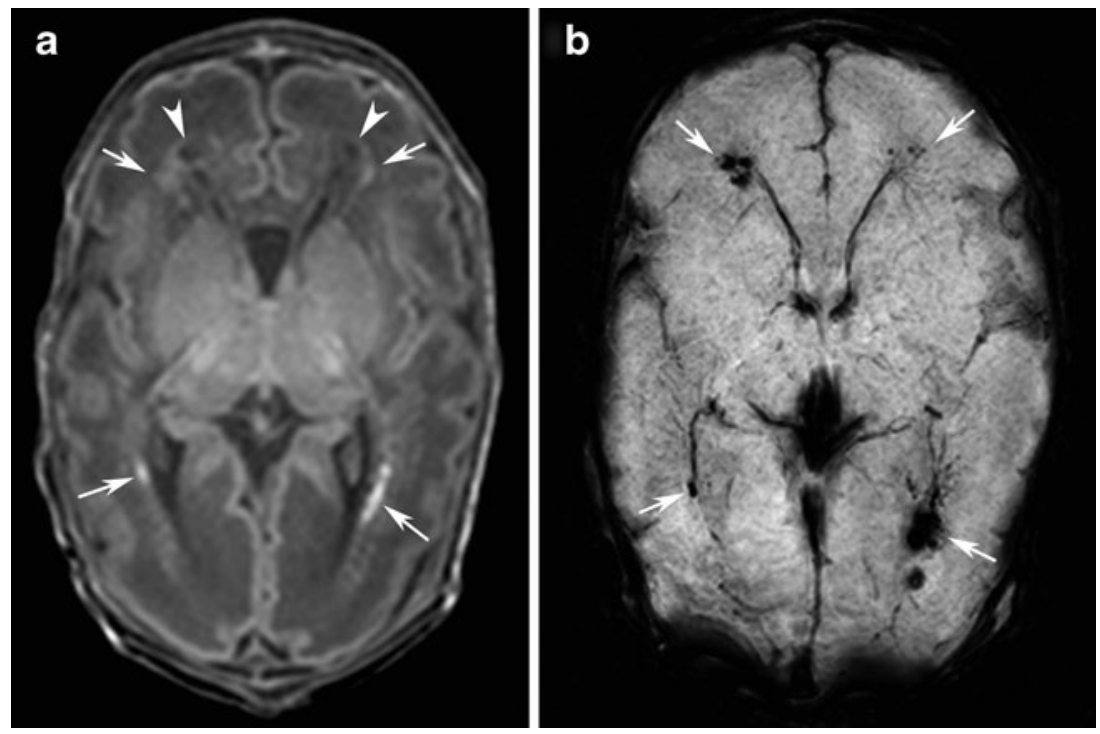
Fig. 5 Postmortem examination in case 23 (same case in Fig. 4). Macroscopic view of the cerebrum on coronal sections A, B. Large cystic areas and hemorrhagic changes can be seen in the white matter $\mathbf{C}$. Low power view of an immunohistological staining using antibodies against CD68 to demonstrate activated microglial cells and macrophages surrounding the cystic and hemorrhagic areas D. GFAP staining is positive in the periventricular area with abnormalities on T1-weighted imaging, but normal SWI ( $\mathbf{E}$ low power and $\mathbf{F}$ high power of central area in $\mathbf{E}$ ), indicating early gliosis. No hemorrhages can be demonstrated in this area
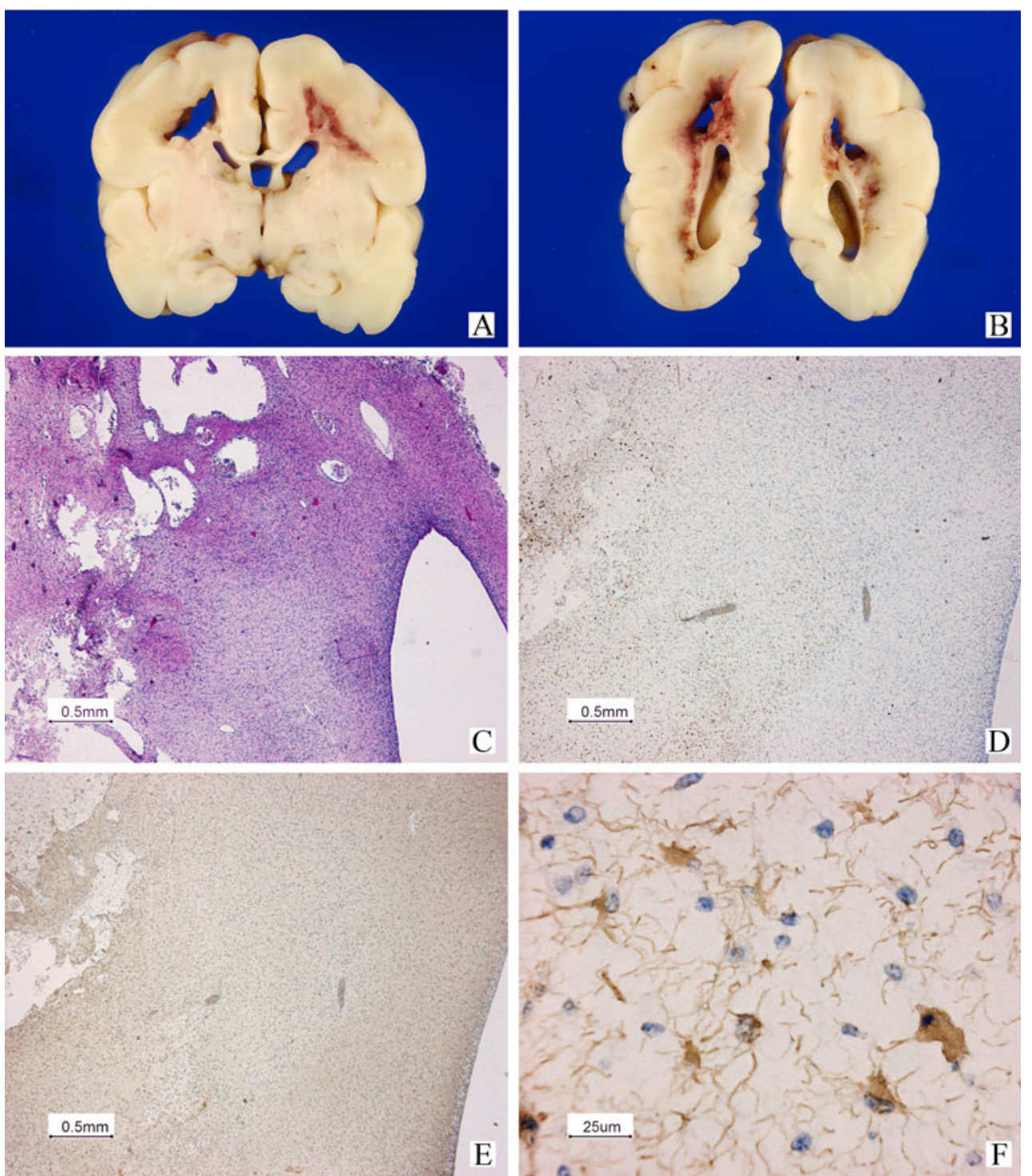

most often considered to be due to early glial scarring or necrosis with mineralization $[1,2,6,8,31,32]$. As most of the infants with PWML survive the neonatal period, histological data coming from postmortem studies are scarce. In our patient with hemorrhagic cystic periventricular leukomalacia, postmortem examination demonstrated early gliosis in the areas with T1-weighted abnormalities, but normal SWI. In the brain areas of T1-weighted and SWI signal loss, hemorrhages could be demonstrated using histology. These findings support the view that some of the T1-weighted abnormalities may be hemorrhages, whereas other lesions may represent early gliosis. Rutherford et al. recently reported postmortem findings in a preterm infant with punctate white matter lesion and found the PWML to correspond to areas of vascular congestion with infiltration of activated microglia [33]. Our results support their findings.
In a recent study by Ramenghi et al. [6], it was shown that the presence of PWML at TEA was associated with a reduced maturation score. Others have shown that the presence of six or more PWML was associated with an increased risk of an adverse neurological outcome [9, 34]. In infants with subtle white matter lesions, a lower percentage of signal loss was found on SWI in PWML, but this percentage tended to increase when the white matter lesions were more severe. These findings suggest that the presence of hemorrhage in PMWL may depend on the underlying etiology of the lesions.

Besides a difference in the presence of signal loss on SWI for the two main patterns of brain injury, it was also of interest to see that PWML, present at TEA, were less often associated with a signal loss on SWI. Previous studies reported that PWML in the preterm period decreased in number or had resolved by TEA $[8,9,34]$. This was in 
agreement with our findings in seven infants who had sequential imaging. The number of PWML on T1-weighted imaging and/or foci of signal loss on SWI suggestive of hemorrhage decreased on follow-up MRI in the seven preterm infants in this study. While some PWML had resolved at TEA, other PWML lesions were still present at the same site on the two sequential MR scans but showed a change in SWI signal intensity, with a low signal on the first MR examination, which was no longer present at TEA. This would suggest that small punctate lesions in preterm infants may resolve relatively fast, whereas other lesions will evolve into early glial scars showing hypersignal on T1-weighted imaging without signal loss on SWI.

Diffusion-weighted image at early MRI showed restricted diffusion in some PWML in this study. Although the etiology of PWML have not been well-established, it is possible that these lesions represent heterogeneous pathology. Some lesions may be related to venous congestion representing small venous infarction with hemorrhage, whereas others may be related to regions of infarction or increased cellularity [33] resulting in gliosis.

In this study, SWI showed various percentage of signal loss in PWML, suggesting various amounts of hemorrhagic components in PWML. Although the nature of these PWML has not been fully explored, SWI can be a very sensitive method to discriminate hemorrhage from other conditions associated with hypersignal on T1-weighted imaging $[35,36]$. Acquisition of SWI was accelerated by EPI, yielding a scan time of $<3$ min for SWI in the current study. Therefore, SWI can be considered as an acceptable additional sequence in infants, which is not time consuming and will provide additional information with regard to ruling out or confirming hemorrhage in lesions with abnormal signal on conventional MRI.

There are several limitations to this study. In the present study, we did not compare SWI findings on 1.5- or 3.0-T systems, which may differ. We aimed to identify all white matter lesions, but small lesions (e.g., smaller than $0.5 \mathrm{~mm}$ ) could not be reliably identified. Comparison of T1weighted (or IR) imaging and SWI may be difficult in very small (e.g., smaller than $1 \mathrm{~mm}$ ) lesions. On the other hand, counting PWML was also difficult in two cases with diffuse white matter hypersignal on T1-weighted image (cases 7 and 23). Because of the magnetic susceptibility effect, signal loss indicating hemorrhage on SWI appears to be bigger, compared to the actual hypersignal area on T1weighted image. Although we considered signal loss larger than the surrounding veins as hemorrhage, complete discrimination between dilated veins and foci of hemorrhage was difficult in cases 7-9. Furthermore, more histopathological studies would be welcome. Another limitation of this study was the relatively small sample size. Follow-up of the infants studied is in progress, and it will be of interest to see whether there is a difference in outcome in those with or without a low signal on SWI within the PWML. Assessment of the prognostic value of SWI using a larger sample size will be of interest.

In conclusion, SWI helps in distinguishing hemorrhagic from non-hemorrhagic PWML. Hemorrhagic PWML are more commonly associated with an intraventricular hemorrhage in the preterm infant, suggesting venous obstruction, while non-hemorrhagic PWML is mainly seen in infants with primarily (subtle) white matter injury, suggesting ischemic or early gliotic lesions. Longitudinal imaging showed a decrease in the number of PWML over time, whereas some PWML became non-hemorrhagic.

Acknowledgments The authors would like to thank our colleagues Thomas Kwee, Vincent Boer, Fredy Visser, and Peter Luijten, Department of Radiology, University Medical Center Utrecht, and Yutaka Imai, Department of Diagnostic Radiology, Tokai University School of Medicine for their valuable remarks, and the technicians for their skillful help.

Conflict of interest We declare that we have no conflict of interest.

Open Access This article is distributed under the terms of the Creative Commons Attribution Noncommercial License which permits any noncommercial use, distribution, and reproduction in any medium, provided the original author(s) and source are credited.

\section{References}

1. Childs AM, Cornette L, Ramenghi LA, Tanner SF, Arthur RJ, Martinez D, Levene MI (2001) Magnetic resonance and cranial ultrasound characteristics of periventricular white matter abnormalities in newborn infants. Clin Radiol 56:647-655

2. Dyet LE, Kennea N, Counsell SJ, Maalouf EF, Ajayi-Obe M, Duggan PJ, Harrison M, Allsop JM, Hajnal J, Herlihy AH, Edwards B, Laroche S, Cowan FM, Rutherford MA, Edwards AD (2006) Natural history of brain lesions in extremely preterm infants studied with serial magnetic resonance imaging from birth and neurodevelopmental assessment. Pediatrics 118:536-548

3. Inder TE, Anderson NJ, Spencer C, Wells S, Volpe JJ (2003) White matter injury in the premature infant: a comparison between serial cranial sonographic and MR findings at term. AJNR Am J Neuroradiol 24:805-809

4. Inder TE, Wells SJ, Mogridge NB, Spencer C, Volpe JJ (2003) Defining the nature of the cerebral abnormalities in the premature infant: a qualitative magnetic resonance imaging study. J Pediatr 143:171-179

5. Leijser LM, de Bruine FT, Steggerda SJ, van der Grond J, Walther FJ, van Wezel-Meijler G (2009) Brain imaging findings in very preterm infants throughout the neonatal period: part I. Incidences and evolution of lesions, comparison between ultrasound and MRI. Early Hum Dev 85:101-109

6. Ramenghi LA, Fumagalli M, Righini A, Bassi L, Groppo M, Parazzini C, Bianchini E, Triulzi F, Mosca F (2007) Magnetic resonance imaging assessment of brain maturation in preterm neonates with punctate white matter lesions. Neuroradiology 49:161-167 
7. Roelants-van Rijn AM, Groenendaal F, Beek FJ, Eken P, van Haastert IC, de Vries LS (2001) Parenchymal brain injury in the preterm infant: comparison of cranial ultrasound, MRI and neurodevelopmental outcome. Neuropediatrics 32:80-89

8. Cornette LG, Tanner SF, Ramenghi LA, Miall LS, Childs AM, Arthur RJ, Martinez D, Levene MI (2002) Magnetic resonance imaging of the infant brain: anatomical characteristics and clinical significance of punctate lesions. Arch Dis Child Fetal Neonatal Ed 86:F171-F177

9. Sie LT, Hart AA, van Hof J, de Groot L, Lems W, Lafeber HN, Valk J, van der Knaap MS (2005) Predictive value of neonatal MRI with respect to late MRI findings and clinical outcome. A study in infants with periventricular densities on neonatal ultrasound. Neuropediatrics 36:78-89

10. Felderhoff-Mueser U, Rutherford MA, Squier WV, Cox P, Maalouf EF, Counsell SJ, Bydder GM, Edwards AD (1999) Relationship between MR imaging and histopathologic findings of the brain in extremely sick preterm infants. AJNR Am J Neuroradiol 20:1349-1357

11. Paneth N, Rudelli R, Monte W, Rodriguez E, Pinto J, Kairam R, Kazam E (1990) White matter necrosis in very low birth weight infants: neuropathologic and ultrasonographic findings in infants surviving six days or longer. J Pediatr 116:975-984

12. Li AM, Chau V, Poskitt KJ, Sargent MA, Lupton BA, Hill A, Roland E, Miller SP (2009) White matter injury in term newborns with neonatal encephalopathy. Pediatr Res 65:85-89

13. Swarte R, Lequin M, Cherian P, Zecic A, van Goudoever J, Govaert P (2009) Imaging patterns of brain injury in term-birth asphyxia. Acta Paediatr 98:586-592

14. Miller SP, McQuillen PS, Hamrick S, Xu D, Glidden DV, Charlton N, Karl T, Azakie A, Ferriero DM, Barkovich AJ, Vigneron DB (2007) Abnormal brain development in newborns with congenital heart disease. N Engl J Med 357:1928-1938

15. Haacke EM, Mittal S, Wu Z, Neelavalli J, Cheng YC (2009) Susceptibility-weighted imaging: technical aspects and clinical applications, part 1. AJNR Am J Neuroradiol 30:19-30

16. Haacke EM, Xu Y, Cheng YC, Reichenbach JR (2004) Susceptibility weighted imaging (SWI). Magn Reson Med 52:612-618

17. Sehgal V, Delproposto Z, Haacke EM, Tong KA, Wycliffe N, Kido DK, Xu Y, Neelavalli J, Haddar D, Reichenbach JR (2005) Clinical applications of neuroimaging with susceptibilityweighted imaging. J Magn Reson Imaging 22:439-450

18. Mori N, Miki Y, Kikuta K, Fushimi Y, Okada T, Urayama S, Sawamoto N, Fukuyama H, Hashimoto N, Togashi K (2008) Microbleeds in moyamoya disease: susceptibility-weighted imaging versus $\mathrm{T} 2 *$-weighted imaging at 3 Tesla. Invest Radiol 43:574-579

19. Nandigam RN, Viswanathan A, Delgado P, Skehan ME, Smith EE, Rosand J, Greenberg SM, Dickerson BC (2009) MR imaging detection of cerebral microbleeds: effect of susceptibilityweighted imaging, section thickness, and field strength. AJNR Am J Neuroradiol 30:338-343

20. Wycliffe ND, Choe J, Holshouser B, Oyoyo UE, Haacke EM, Kido DK (2004) Reliability in detection of hemorrhage in acute stroke by a new three-dimensional gradient recalled echo susceptibility-weighted imaging technique compared to computed tomography: a retrospective study. J Magn Reson Imaging 20:372-377
21. Groenendaal F, Leusink C, Nijenhuis M, Janssen MJ (2002) Neonatal life support during magnetic resonance imaging. J Med Eng Technol 26:71-74

22. Cowan FM (1998) Sedation for magnetic resonance scanning of infants and young children. Principles and practice of sedation. Blackwell Heathcase, London

23. Rauscher A, Sedlacik J, Barth M, Mentzel HJ, Reichenbach JR (2005) Magnetic susceptibility-weighted MR phase imaging of the human brain. AJNR Am J Neuroradiol 26:736-742

24. Papile LA, Burstein J, Burstein R, Koffler H (1978) Incidence and evolution of subependymal and intraventricular hemorrhage: a study of infants with birth weights less than 1,500 gm. J Pediatr 92:529-534

25. de Vries LS, Eken P, Dubowitz LM (1992) The spectrum of leukomalacia using cranial ultrasound. Behav Brain Res 49:1-6

26. de Vries LS, Roelants-van Rijn AM, Rademaker KJ, Van Haastert IC, Beek FJ, Groenendaal F (2001) Unilateral parenchymal haemorrhagic infarction in the preterm infant. Eur $\mathrm{J}$ Paediatr Neurol 5:139-149

27. Perlman JM (1998) White matter injury in the preterm infant: an important determination of abnormal neurodevelopment outcome. Early Hum Dev 53:99-120

28. Childs AM, Ramenghi LA, Cornette L, Tanner SF, Arthur RJ, Martinez D, Levene MI (2001) Cerebral maturation in premature infants: quantitative assessment using MR imaging. AJNR Am J Neuroradiol 22:1577-1582

29. Counsell SJ, Maalouf EF, Fletcher AM, Duggan P, Battin M, Lewis HJ, Herlihy AH, Edwards AD, Bydder GM, Rutherford MA (2002) MR imaging assessment of myelination in the very preterm brain. AJNR Am J Neuroradiol 23:872-881

30. Groenendaal F, Lammers H, Smit D, Nikkels PG (2006) Nitrotyrosine in brain tissue of neonates after perinatal asphyxia. Arch Dis Child Fetal Neonatal Ed 91(6):F429-F433. doi:10.1136/ adc. 2005.092114

31. Sie LT, van der Knaap MS, van Wezel-Meijler G, Taets van Amerongen AH, Lafeber HN, Valk J (2000) Early MR features of hypoxic-ischemic brain injury in neonates with periventricular densities on sonograms. AJNR Am J Neuroradiol 21:852861

32. Volpe JJ (2001) Neurology of the newborn, 4th edn. WB Saunders, Philadelphia

33. Rutherford MA, Supramaniam V, Ederies A, Chew A, Bassi L, Groppo M, Anjari M, Counsell S, Ramenghi LA (2010) Magnetic resonance imaging of white matter diseases of prematurity. Neuroradiology 52(6):505-521

34. Miller SP, Cozzio CC, Goldstein RB, Ferriero DM, Partridge JC, Vigneron DB, Barkovich AJ (2003) Comparing the diagnosis of white matter injury in premature newborns with serial MR imaging and transfontanel ultrasonography findings. AJNR Am J Neuroradiol 24:1661-1669

35. Kesavadas C, Santhosh K, Thomas B, Gupta AK, Kapilamoorthy TR, Bodhey N, Pendharker H, Patro S (2009) Signal changes in cortical laminar necrosis-evidence from susceptibility-weighted magnetic resonance imaging. Neuroradiology 51:293-298

36. Niwa T, Aida N, Shishikura A, Fujita K, Inoue T (2008) Susceptibility-weighted imaging findings of cortical laminar necrosis in pediatric patients. AJNR Am J Neuroradiol 29:17951798 